Diabetologe $2021 \cdot 17: 819-821$

https://doi.org/10.1007/s11428-021-00831-y

Angenommen: 28. Oktober 2021

Online publiziert: 15 . November 2021

(c) Der/die Autor(en) 2021

\section{Von glukozentrischen zu kardio- und renozentrischen Behandlungsoptionen bei Typ-2-Diabetes}

\author{
Rima Chakaroun ${ }^{1,2}$ \\ 'Klinik und Poliklinik für Endokrinologie, Nephrologie, Rheumatologie, Universitätsklinikum Leipzig, \\ Leipzig, Deutschland \\ ${ }^{2}$ Wallenberg Laboratory, Universität Göteburg, Göteburg, Schweden
}

\section{Originalpublikation}

Hertzel CG, Sattar N, Rosenstock J et al for the AMPLITUDE-O Trial Investigators (2021) Cardiovascular and renal outcomes with efpeglenatide in type 2 diabetes. $N$ Engl J Med 385(10):896-907. https://doi.org/10.1056/ NEJMoa2108269

Hintergrund. Laut aktueller Projektionen wird die Anzahl der Menschen, die an Typ2-Diabetes (T2D) leiden, zwischen 2019 und 2045 von 463 Mio. auf 700 Mio. weltweit steigen [12]. Hiermit verbunden ist ein erwarteter Anstieg der kardiovaskulären Morbidität und Mortalität [4]. In Industrieländern ist T2D zudem die häufigste Ursache einer chronischen und terminalen Niereninsuffizienz (CKD, [7]). Auch steigt die Wahrscheinlichkeit kardiovaskulärer Ereignisse mit einer Verschlechterung der glykämischen Kontrolle und der Nierenfunktion [3, 9].

Mehrere Glukagon-ähnliches-Peptid-1Rezeptor-Agonisten (GLP-1-RA), die dem menschlichen GLP-1 strukturell ähneln, reduzieren nachweislich das Risiko kardiovaskulärer Ereignisse bei T2D [13]. Mit der zunehmenden Integration kardio- und renoprotektiver Medikation in aktuelle Therapieschemata stellt sich die Frage, ob es zu einem Sättigungseffekt kommt oder ob Patienten mit fortgeschrittener kardiovaskulärer Morbidität von einer Kombination von GLP-1-RA beispielsweise mit SGLT-2Hemmern (sodium-glucose linked transporter 2) weiter profitieren können. Bisher hatten Endpunktstudien mit GLP-1-RA keine repräsentativen Subgruppen, um diese Frage zu beantworten. Efpeglenatid ist ein weiterer GLP-1-RA, der auf dem Exendin-4-Molekül basiert und unter dem eine Senkung der Blutglukosewerte ohne $\mathrm{Hy}$ poglykämien nachgewiesen wurde [11]. Seine Auswirkungen auf kardiovaskuläre oder renale Endpunkte bei Hochrisikopatienten mit T2D waren bislang unklar.

Methoden. Bei der AMPLITUDE-O-Studie handelt es sich um eine internationale, randomisierte, placebokontrollierte Phase-3Studie, die die Wirksamkeit und Sicherheit von Efpeglenatid bei Patienten mit T2D in 28 Ländern zwischen Mai 2018 und April 2019 untersuchte. Rekrutiert wurden insgesamt 4076 Patienten (mittleres Alter 64,5 Jahre) mit T2D, bei denen eine kardiovaskuläre Erkrankung bereits vorlag oder eine Nierenfunktionseinschränkung (eGRF-Werte zwischen 25,0 und $59,9 \mathrm{ml} / \mathrm{min} / 1,73 \mathrm{~m}^{2}$ ) sowie mindestens ein weiterer kardiovaskulärer Risikofaktor bekannt waren. Es erfolgte eine 1:1:1Randomisierung, in der insgesamt 2717 Studienteilnehmer Efpeglenatid in einer Dosierung von 4 oder $6 \mathrm{mg}$ per subkutaner Injektion wöchentlich erhielten und 1359 der Placebogruppe zugeteilt wurden, zusätzlich zu ihren aktuellen Behandlungsschemata. Der primäre Endpunkt war das erste schwerwiegende unerwünschte kardiovaskuläre Ereignis, zusammengesetzt aus nichttödlichem Myokardinfarkt, nichttödlichem Schlaganfall oder Tod aufgrund von kardiovaskulären oder unbestimmten Ursachen.

Der sekundäre Endpunkt fasste als Komponenten eine neu aufgetretene Makroal- 
buminurie, eine anhaltende Abnahme der Nierenfunktion (eGRF-Abnahme $\geq 40 \%$ über mehr als 30 Tage, renale Ersatztherapie für mehr als 90 Tage oder anhaltend niedrige eGFR-Werte $<15 \mathrm{ml} / \mathrm{min} / 1,73 \mathrm{~m}^{2}$ ) zusammen. Eine Reihe weiterer Endpunkte bezogen auf Ereignisse, Hospitalisierung, Gewicht, glykämische Kontrolle wurden eingeschlossen.

Ergebnisse. Zu Studienbeginn wiesen knapp $90 \%$ der Studienteilnehmer eine manifeste kardiovaskuläre Erkrankung und $32 \%$ eine eingeschränkte Nierenfunktion auf. Bei einer medianen Beobachtungszeit von 1,8 Jahren trat der primäre Endpunkt bei $7 \%$ in der Efpeglenatid- vs. 9,2\% in der Placebogruppe ein („hazard ratio" [HR]: 0,73, $95 \%$-Konfidenzintervall [95\%KI]: 0,58-0,92), womit sowohl die Nichtunterlegenheit $(p<0,001)$ als auch die Überlegenheit $(p=0,007)$ gegenüber Placebo belegt wurden. Dieser Benefit betraf auch die Einzelkomponenten des Endpunktes (nichttödliche Myokardinfarkte $[3,1 \%$ vs. $3,9 \%]$, Schlaganfälle $[1,5 \%$ vs. $1,8 \%]$, kardiovaskuläre Mortalität [2,8\% vs. 3,7\%]). Es müssen somit 46 Patienten behandelt werden, um ein kardiovaskuläres Ereignis zu verhindern. Zum Vergleich liegt diese Zahl bei 49 für Statine für die Prävention eines Herzinfarkts und 155 für die Prävention eines Schlaganfalls [14]. Die Inzidenzrate für den kombinierten renalen Endpunkt war 32\% niedriger unter Efpeglenatid als unter Placebo (13\% vs. $18,4 \%$, HR: 0,$68 ; 95 \%-K l: 0,57-0,79$; $p<0,001)$. Vermutlich am interessantesten war der kardiovaskuläre und renale Benefit unabhängig von der gleichzeitigen Behandlung mit SGLT-2-Hemmern (Einsatz bei $15 \%$ der Kohorte) und somit additiv. Bezüglich Sicherheit ist das Profil von Efpeglenatid vergleichbar mit dem anderer GLP-1-RA, womit gastrointestinale Beschwerden (Übelkeit, Diarrhö, Obstipation, Blähung und Erbrechen) signifikant häufiger in der Behandlung- als in der Placebogruppe auftraten (1,2\% vs. $0,4 \%, p=0,03)$.

\section{Zusammenfassung und Kommentar}

Basierend auf einer Reihe von Outcomestudien wurde der Einsatz von GLP-1-RA und
SGLT-2-Hemmern in den jüngsten Leitlinien endokrinologischer und kardiologischer Gesellschaften verankert [2, 5]. Diese tragen, unabhängig von ihren Effekten auf die Blutglukose, zu einer merklichen Reduktion kardiovaskulärer Mortalität und Morbidität bei. Mit Efpeglenatid reihte sich nun der 5. GLP-1-RA neben Liraglutid, Albiglutid, Semaglutid und Dulaglutid ein. Man könne sich natürlich fragen: „Warum noch ein GLP-1-RA?". Frühere Daten deuteten darauf hin, dass die kardiovaskulären Vorteile von GLP-1-Rezeptor-Agonisten eher auf humanbasierte als auf exendinbasierte GLP-1-RA beschränkt sein könnten [8].

Aber auch für das exendinbasierte Efpeglenatid zeigte sich eine Reduktion des zusammengesetzten kardiovaskulären Endpunkts um 27\%. Eine aktuelle Metaanalyse belegte, unter Hinzunahme der Ergebnisse der AMPLITUDE-O-Studie, dass keine statistische Heterogenität der kardiovaskulären Wirksamkeit durch die molekulare Struktur des GLP-1-RezeptorAgonisten besteht, womit es sich am ehesten um einen Effekt der Medikamentenklasse handelt [13].

Die additive Senkung des kardiovaskulären Risikos durch GLP-1-RA unabhängig von einer gleichzeitigen Therapie mit einem SGLT-2-Hemmer ist eine Haupterkenntnis der AMPLITUDE-O-Studie.

Die AMPLITUDE-O-Studie zeigte zudem neben "harmony outcomes" eine signifikante Reduktion der Hospitalisierungsrate aufgrund einer dekompensierten Herzinsuffizienz. Zwei Studien, die die Wirkung von Liraglutid vs. Placebo bei Patienten mit Herzinsuffizienz und reduzierter Ejektionsfraktion mit oder ohne T2D untersuchten, ergaben jedoch eher ungünstige Risikoeinschätzungen [6, 10], sodass eine Extrapolation und der Einsatz von GLP-1RA bei Herzinsuffizienz mit eingeschränkter Ejektionsfraktion mit Vorsicht zu genießen sind.

Die Limitation dieser Studie gelten für alle anderen GLP-1-RA-Studien: Die Daten zur kardiovaskulären Wirksamkeit von GLP-1-RA in der primären kardiovaskulären Prävention sind noch begrenzt. Auch ist die mediane Beobachtungszeit kurz, sodass längerfristige Effekte eher unbekannt bleiben. Unabhängig hiervon besteht weiterhin eine große Unsicherheit hinsicht- lich der Wirkungsmechanismen, die zu den kardiovaskulären Effekten dieser Medikamentenklasse führen und die unabhängig von ihren Effekten auf glykometabolische Parameter zu sein scheinen.

Auch wenn die Studien überzeugen, wird für Efpeglenatid - wie für andere GLP 1-RA - eine große Diskrepanz zwischen wissenschaftlicher und klinischer „Begeisterung" erwartet. Erklärend für den zurückhaltenden Einsatz ist zweifellos die Überteuerung dieser Präparate, die hiermit für einen großen Teil der Patienten mit T2D unzugänglich sind. Mit zunehmender Verfügbarkeit neuer "smart drugs" [1] besteht jedoch die Möglichkeit, maßgeschneiderte medikamentöse Therapieregime anzubieten, was den Einsatz dieser hocheffektiven polypotenten Präparate motivieren kann und soll.

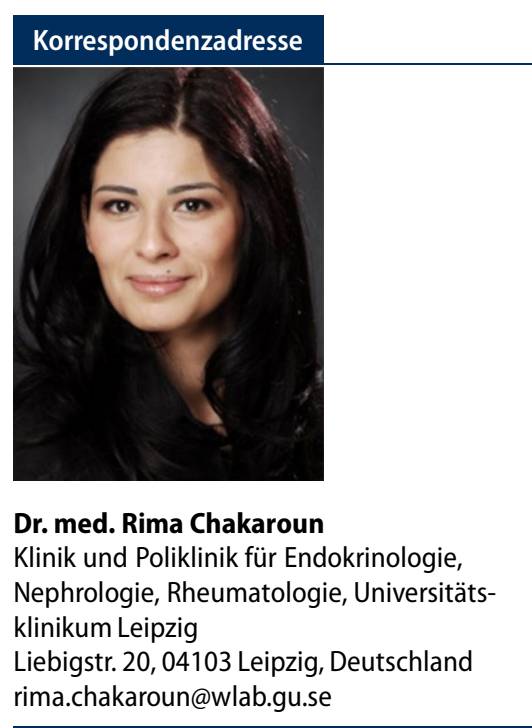

Funding. Open access funding provided by University of Gothenburg.

Interessenkonflikt. R. Chakaroun gibt an, dass kein Interessenkonflikt besteht.

Open Access. Dieser Artikel wird unter der Creative Commons Namensnennung 4.0 International Lizenz veröffentlicht, welche die Nutzung, Vervielfältigung, Bearbeitung, Verbreitung und Wiedergabe in jeglichem Medium und Format erlaubt, sofern Sie den/die ursprünglichen Autor(en) und die Quelle ordnungsgemäß nennen, einen Link zur Creative Commons Lizenz beifügen und angeben, ob Änderungen vorgenommen wurden.

Die in diesem Artikel enthaltenen Bilder und sonstiges Drittmaterial unterliegen ebenfalls der genannten Creative Commons Lizenz, sofern sich aus der Abbildungslegende nichts anderes ergibt. Sofern das be- 
treffende Material nicht unter der genannten Creative Commons Lizenz steht und die betreffende Handlung nicht nach gesetzlichen Vorschriften erlaubt ist, ist für die oben aufgeführten Weiterverwendungen des Materials die Einwilligung des jeweiligen Rechteinhabers einzuholen.

Weitere Details zur Lizenz entnehmen Sie bitte der Lizenzinformation auf http://creativecommons.org/ licenses/by/4.0/deed.de.

\section{Literatur}

1. Bailey CJ (2021) Tirzepatide: a new low for bodyweight and blood glucose. Lancet Diabetes Endocrinol 9:646-648

2. Buse JB, Wexler DJ, Tsapas A et al (2020) 2019 update to: management of hyperglycemia in type 2 diabetes, 2018. A consensus report by the American diabetes association (ADA) and the European association for the study of diabetes (EASD). Diabetes Care 43:487-493. https://doi. org/10.2337/dci19-0066

3. Cherney DZI, Repetto E, Wheeler DC et al (2020) Impact of cardio-renal-metabolic comorbidities on cardiovascular outcomes and mortality in type 2 diabetes mellitus. Am J Nephrol 51:74-82

4. Dinesh Shah A, Langenberg C, Rapsomaniki E et al (2015) Type 2 diabetes and incidence of a wide range of cardiovascular diseases: a cohort study in 1.9 million people. Lancet 385(1):S86

5. Grant PJ, Cosentino F (2019) The 2019 ESC guidelines on diabetes, pre-diabetes, and cardiovascular diseases developed in collaboration with theEASD: new features and the ten commandments' of the 2019 guidelines are discussed by professor Peter J. Grant and professor Francesco Cosentino, the task force chairmen. Eur Heart J 40:3215-3217

6. Jorsal A, Kistorp C, Holmager P et al (2017) Effect of liraglutide, a glucagon-like peptide-1 analogue, on left ventricular function in stable chronic heart failure patients with and without diabetes ( LIVE ) - a multicentre, double-blind, randomised placebo-controlled trial. Eur J Heart Fail 19:69-77. https://doi.org/10.1002/ejhf.657

7. Koye DN, Magliano DJ, Nelson RG, Pavkov ME (2018) The global epidemiology of diabetes and kidney disease. Adv Chronic Kidney Dis 25:121-132

8. Kristensen SL, Rørth R, Jhund PS et al (2019) Cardiovascular, mortality, and kidney outcomes with GLP-1 receptor agonists in patients with type 2 diabetes: a systematic review and meta-analysis of cardiovascular outcome trials. Lancet Diabetes Endocrinol 7:776-785. https://doi.org/10.1016/ s2213-8587(19)30249-9

9. Laiteerapong N, Ham SA, Gao Y et al (2019) The legacy effect in type 2 diabetes: impact of early glycemic control on future complications (the diabetes \& aging study). Diabetes Care 42:416-426

10. Margulies KB, Hernandez AF, Redfield MM et al (2016) Effects of liraglutide on clinical stability among patients with advanced heart failure and reduced ejection fraction. JAMA 316:500. https:// doi.org/10.1001/jama.2016.10260

11. Rosenstock J, Sorli CH, Trautmann ME et al (2019) Once-weekly efpeglenatide dose-range effects on glycemic control and body weight in patients with type 2 diabetes on metformin or drug naive, referenced to liraglutide. Diabetes Care 42:1733-1741

12. Saeedi P, Petersohn I, Salpea P et al (2019) Global and regional diabetes prevalence estimates for 2019 and projections for 2030 and 2045: results from the international diabetes federation diabetes atlas, 9th edition. Diabetes Res Clin Pract 157:107843. https://doi.org/10.1016/j.diabres. 2019.107843

13. Sattar N, Lee MMY, Kristensen SL et al (2021) Cardiovascular, mortality, and kidney outcomes with GLP-1 receptor agonists in patients with type 2 diabetes: a systematic review and meta-analysis of randomised trials. Lancet Diabetes Endocrinol. https://doi.org/10.1016/S2213-8587(21)00203-5

14. Yerrakalva D, Griffin SJ (2017) Statins for primary prevention in people with a 10\% 10-year cardiovascular risk: too much medicine too soon? BrJGen Pract 67:40-41

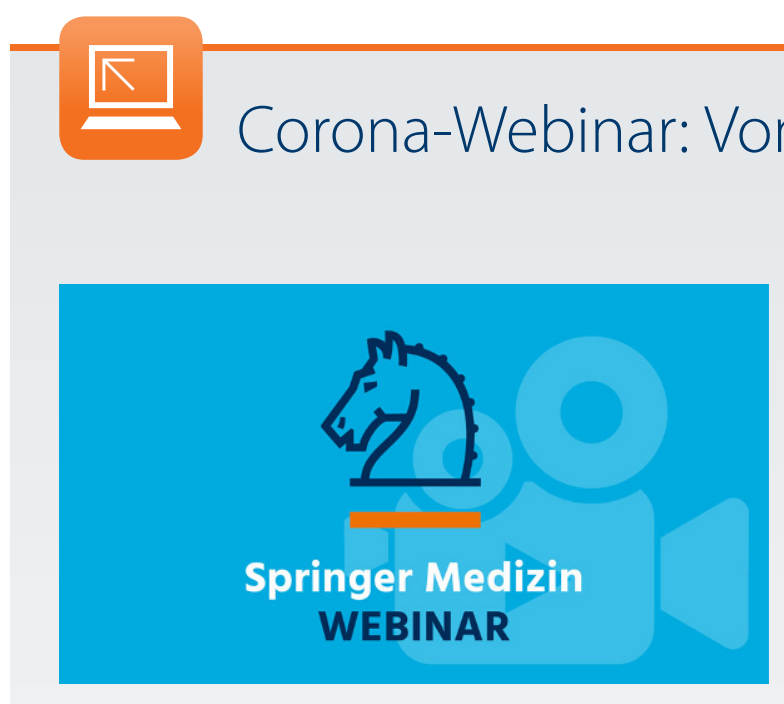

Boostern, Impfquoten, Durchbruchinfektionen: Auch in der vierten Welle der Coronapandemie steht die Vakzine im Fokus, denn „Bei der Therapie sind wir noch längst nicht so weit, wie wir sein wollen." Prof. Bernd Salzberger blickt im Webinar auf die für den kommenden Coronawinter relevanten Studiendaten - und die Lage in den Kliniken: Wer kommt ins Krankenhaus, wer liegt auf Intensiv und wer verstirbt? Die Empfehlungen des Infektiologen für das Vorgehen in den nächsten Wochen sind klar.
Der Referent: Prof. Dr. Bernd Salzberger

- Bereichsleiter Infektiologie, Abteilung für Krankenhaushygiene

und Infektiologie am Universitätsklinikum Regensburg

- Präsident der Deutschen Gesellschaft für Infektiologie (DGI).

- Langjähriger Herausgeber der Zeitschrift Der Internist mit besonderem Fokus auf die Betreuung infektiologischer Themen

Das Video und alle Empfehlungen finden Sie nach Registrierung kostenfrei hier:

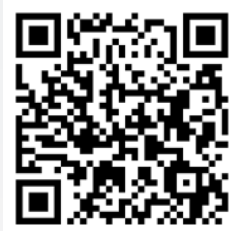

https://www.springermedizin.de/link/19836182 